\title{
Bajc-Melfo vacua enable Yukawon ultraminimal grand unified theories
}

\author{
Charanjit S. Aulakh" \\ Indian Institute of Science Education and Research Mohali, \\ Sector 81, S. A. S. Nagar, Manauli 140306, India \\ (Received 1 July 2014; published 11 March 2015)
}

\begin{abstract}
Bajc-Melfo (BM) two-field $(S, \phi)$ superpotentials define metastable F-term supersymmetry-breaking vacua suitable as hidden sectors for calculable and realistic family and grand unification models. The undetermined vacuum expectation value (VEV) $\left\langle S_{s}\right\rangle$ of the Polonyi field that breaks supersymmetry can be fixed either by coupling to $N=1$ supergravity or by radiative corrections. BM hidden sectors extend to symmetric multiplets $(S, \phi)_{a b}$ of a gauged $O\left(N_{g}\right)$ family symmetry, broken at the GUT scale, so that the $O\left(N_{g}\right)$ charged component VEVs $\left\langle\hat{S}_{a b}\right\rangle$ are also undetermined before accounting for the $O\left(N_{g}\right)$ D-terms, which fix them by cancellation against D-term contributions from the visible sector. This facilitates Yukawon ultraminimal GUTs (YUMGUTs) proposed in [C. S. Aulakh and C. K. Khosa, Phys. Rev. D 90, 045008 (2014)] by relieving the visible sector from the need to give null D-terms for the family symmetry $O\left(N_{g}\right)$. We analyze symmetry breaking and spectra of the hidden-sector fields in the supergravity resolved case when $N_{g}=1,2,3$. Besides the Polonyi field $S_{s}$, most of the superfields $\hat{S}_{a b}$ remain light, with fermions getting masses only from loop corrections. Such modes may yield novel dark matter lighter than $100 \mathrm{GeV}$. Possible Polonyi and moduli problems associated with the fields $S_{a b}$ call for detailed investigation of loop effects due to the Yukawa and gauge interactions in the hidden sector and of postinflationary field relaxation dynamics.
\end{abstract}

DOI: 10.1103/PhysRevD.91.055012

PACS numbers: 12.10.Dm, 11.30.Hv

\section{INTRODUCTION}

Supersymmetry (SUSY) imposes remarkable restrictions on its own spontaneous violation and thus severely constrains models of physics beyond the standard model. Traditionally [1-5] gravity mediation has been used to generate phenomenologically acceptable soft SUSYbreaking terms for globally supersymmetric GUTs. This requires supersymmetry breaking in a hidden sector with no superpotential couplings to the "visible" sector in which we live. The GUT Minkowski space vacuum is metastable [4] in the sense of there being a SUSY-preserving vacuum with lower (negative) energy, but it is thought that it cannot decay to the lower vacuum by tunneling, or at any rate the time scale for doing so is much larger than the age of the Universe. The vacuum in the gravity mediated scenario is defined as the perturbation of a global SUSY-preserving minimum by a hidden sector with a global minimum which breaks supersymmetry spontaneously due to specialized superpotentials (Polonyi, O'Raifeartaigh [6,7], etc.) or due to strong dynamics (gaugino condensation, etc.). After long struggles to tame the hard problem of finding

\footnotetext{
*aulakh@pu.ac.in; aulakh@iisermohali.ac.in; http://14.139.227. 202/Faculty/aulakh/

Published by the American Physical Society under the terms of the Creative Commons Attribution 3.0 License. Further distribution of this work must maintain attribution to the author $(s)$ and the published article's title, journal citation, and DOI.
}

phenomenologically usable SUSY-breaking global minima, it was reemphasized $[8,9]$ that metastable, i.e. local, minima could serve just as well as global minima, since the unifying theory would anyway have vacua at lower energy [4] than the phenomenologically relevant Minkowski vacuum on which the MSSM lived. This realization was liberating for phenomenologists, because models defined on supersymmetry-breaking vacua which are only local minima offer a much larger range of candidate vacua. Various strongly coupled supersymmetric gauge theories $[9,10]$ were studied to provide the SUSY-breaking dynamics, albeit now considering also metastable vacua. Unfortunately, even the metastable dynamical SUSYbreaking models seem so arbitrarily elaborate as to rival the complexity of the phenomenological theory (GUT, MSSM, etc.) for which they were supposed to provide the service of supersymmetry breaking, making testability of both a moot issue.

Recently Bajc and Melfo (BM) [11] suggested that, from a "calculable unification" viewpoint, it would be more interesting to consider SUSY-breaking metastable vacua of simple two-field hidden-sector superpotentials. BM models are related to the classic O'Raifeartaigh SUSY-breaking superpotentials and are variants of a class of models with SUSY-breaking local minima studied systematically earlier in Ref. [12]. Such superpotentials typically have flat directions, and there is a longstanding vision whereby the determination of such flat directions (running out of SUSY-breaking minima) by radiative corrections [13] or 
supergravity $[14,15]$ might profitably fix the undetermined vacuum expectation values (VEVs) at a high unification scale. Bajc and Melfo took up this challenge and formulated models where the undetermined VEVs were those of 24 or 75 irreps of an $S U(5)$ unified theory and the flat directions were determined by radiative corrections. The use of of $N=1$ supergravity potentials to lift the flat directions rolling out of the Bajc-Melfo SUSY-breaking local minimum has not been analyzed.

From a Grand Unified viewpoint, the only credible sign of flavor unification so far is third-family Yukawa unification at large $\tan \beta$ in $S O(10)$ at scales of order $M_{X}>10^{16} \mathrm{GeV}$. On the other hand, minimal supersymmetric (MS) $S O(10)$ GUTs [16-23] have been shown to be completely realistic and even to suppress $d=5$ proton decay $[24,25]$ by a novel and generic mechanism based on the necessary emergence of a pair of light doublet Higgs in the effective MSSM. Motivated by these two clues, we exploited the defining (i.e. $\overline{\mathbf{1 2 6}}$ ) representation of MSGUTs (which gets large VEVs yet couples to matter fermions) to design a renormalizable Grand Unified Yukawon/ familion model [26] in which we generate hierarchical matter fermion Yukawas from flavor-bland parameters of the MSGUT extended by an $O\left(N_{g}\right)$ family symmetry. Family gauge symmetry is broken at the unification scale, and the visible-sector GUT Higgs fields effloresce into symmetric $(\mathbf{2 1 0}, \mathbf{1 2 6}, \overline{\mathbf{1 2 6}}, \mathbf{1 0})$ or antisymmetric $(\mathbf{1 2 0})$ irreps of the family group [as dictated by the properties of bilinears in the 16-plet matter irreps in $S O(10)$ GUTs], while the matter 16-plet Yukawa couplings become flavor bland. The neat and exactly soluble [18] gauge symmetry breaking $[S O(10) \rightarrow$ MSSM $]$ of the family singlet MSGUT Higgs system then generalizes straightforwardly for the family symmetry variant MSGUT Higgs multiplets. The supersymmetric breaking of family symmetry will be discussed in detail in this paper. Extraction of the light MSSM Higgs from the plethora of MSSM doublets present in the $O\left(N_{g}\right) \times S O(10)$ GUT via calculation of the "Higgs fractions" $[18,20,23,27]$ (which specify the makeup of the MSSM Higgs) determined by flavor-blind GUT parameters generates candidate flavor structures.

In implementing this flavor-generation scenario an obstacle arises. Although the solution of the F- and Dterm conditions for the GUT multiplets in the $S O(10)$ sector with flavor nondiagonal VEVs is straightforward, it is very hard or impossible to ensure that the same configurations simultaneously cancel the family symmetry D-terms. It thus becomes necessary to introduce additional fields to soak up the contribution of the GUT VEVs to the $O\left(N_{g}\right)$ D-terms. Since minimality, simplicity and solubility of the GUT Higgs sector symmetry breaking is a central virtue of MSGUTs, it is natural to ask whether the additional fields might not be located in a hidden sector with its own flat directions arising from supersymmetry breaking in a way which did not interfere with the already accomplished MSGUT symmetry breaking [16-18]. Besides making a completely unexpected connection between family symmetry and supersymmetry breaking, this idea is straightforward to implement in a context where the BM flat directions are lifted by a combination of supergravity and $O\left(N_{g}\right)$ gauge D-term minimization effects.

A notable phenomenological implication of our model is the presence of weakly coupled light fields ("moduli"). In particular, in the supergravity mediated scenario presented here, there is a Polonyi (scalar) field (Planck-scale VEV, weak-scale mass and intermediate-scale SUSY-breaking F-term). In addition, there are other light fermions and scalars associated with the flat directions of the family nonsinglet BM fields. These modes may be interesting light $(<50 \mathrm{GeV})$ SUSY WIMP dark matter of the sort perhaps indicated by the DAMA/LIBRA experiment [28] and so far completely missing from MSGUT spectra. On the other hand, this clutch of modes may also pose the typical problems associated with "moduli" fields found in other fundamental theories [29]. Recall that the Polonyi problem arises due to late decay of the oscillations of the supersymmetry-breaking Polonyi field while it settles into the zero-temperature potential's minimum because of the suppression of its couplings by the Planck scale. The dark matter candidates' role in cosmogony also needs to be checked for consistency. In view of the new Yukawa and gauge interactions of the hidden sector, the cosmological implications require a separate detailed study and thus should not prematurely prejudice the novel flavor-generation aspects of our model. In this paper we give the relevant details for the symmetry breaking, VEVs and masses in the BM sector for direct use in our "Yukawonification" [26] models. We will return to a study of cosmological constraints and alternative models elsewhere.

\section{THE BAJC MELFO VACUA AND SUPERGRAVITY}

The Bajc-Melfo superpotential [11] is a quadratic variant of of the O'Raifeartaigh superpotential and its relatives $[7,12]$ which breaks superymmetry at a metastable minimum

$$
W_{\mathrm{BM}}\left(S_{s}, \phi_{s}\right)=W_{0}+S_{s}\left(\mu_{B} \phi_{s}+\lambda_{B} \phi_{s}^{2}\right) .
$$

We have added a constant term $W_{0}$ for later convenience. It is then easy to see $[11,12]$ that besides the SUSYpreserving global minima at $S_{s}=\phi_{s}=0$ and $S_{s}=0$, $\phi_{s}=-\mu_{B} / \lambda_{B}$ with zero vacuum energy, there is also a local minimum at $\left\langle\phi_{s}\right\rangle=-\mu_{B} / 2 \lambda_{B}$ with $\left\langle S_{s}\right\rangle$ undetermined provided

$$
\left|\left\langle S_{s}\right\rangle\right| \geq\left|\frac{\left\langle\phi_{s}\right\rangle}{\sqrt{2}}\right|
$$


This local minimum breaks supersymmetry, since $\left\langle F_{S}\right\rangle=$ $-\mu_{B}^{2} / 4 \lambda_{B} \equiv \theta$. Thus, the chiral fermion in the multiplet $S_{s}$ provides the Goldstino field, while its partner scalar, whose VEV remains undetermined, is massless. When supersymmetry is made local by coupling the theory to gravity, the Goldstino can be gauged away by a local supersymmetry transformation, leaving a massive gravitino while the scalar picks up the gravitino mass common to light scalars in gravity mediation. For clarity we first review the effect of coupling to supergravity on extrema of globally supersymmetric Lagrangians [4].

The generic $N=1$ supergravity potential $[1,3,5]$ for scalar fields $z_{I}$ interacting through a superpotential $W\left(z_{I}\right)$ [with canonical Kahler potential $K(z, \bar{z})$ and gauge kinetic function $f_{\alpha \beta}(z)=\delta_{\alpha \beta}, \kappa$ is the Planck length] is

$$
\begin{aligned}
V= & E\left(\sum_{I}\left|\mathcal{F}^{I}\right|^{2}-3 \kappa^{2}|W|^{2}\right) \\
& +\sum_{\alpha} \frac{g_{\alpha}^{2}}{4}\left[\frac{\left(\mathcal{F}^{I}\left(T^{\alpha}\right)_{I}^{J} z_{J}\right)^{2}}{\kappa^{4} W^{2}}+\text { H.c. }\right], \\
\mathcal{F}^{I}= & \frac{\partial W}{\partial z_{I}}+z^{* I} \kappa^{2} W ; \quad E \equiv e^{\kappa^{2} \sum_{I}\left|z_{I}\right|^{2}} .
\end{aligned}
$$

As is well known, the constant term in the superpotential $W$, which is irrelevant in global supersymmetry, can be used to tune the vacuum energy in one of its extrema to zero after coupling to supergravity. This means that for a supersymmetric minimum with $\operatorname{VEVs}\left\langle z_{I}\right\rangle=\bar{z}_{I}$ derived from

$$
\left\langle F^{I}\right\rangle=\left\langle\partial W / \partial z_{I}\right\rangle=0 ; \quad\left\langle D^{\alpha}\right\rangle=\left\langle z^{* I} T_{I}^{\alpha J} z_{J}\right\rangle=0
$$

there is no shift in the global VEVs $\left\langle\bar{z}_{I}\right\rangle$ at all: since by tuning $\langle W\rangle=\bar{W}=0$, we ensure that the $\operatorname{VEV} \overline{\mathcal{F}}^{I}=\bar{F}^{I}=0$, the vacuum energy at the old minimum vanishes, and so do terms in the extremization condition which come from the variations of the fields in the exponential factor (since they multiply a separately vanishing factor). It is, however, amusing that the D-term VEV in (3) above is then indeterminate, seemingly indicating an incompatibility between supergravity and preserved supersymmetry with $\langle W\rangle=0$.

On the other hand, if the global superpotential breaks supersymmetry spontaneously (as, for example, the Polonyi or O'Raifeartaigh superpotentials at a global minimum or the BM superpotential at a local minimum), then the nonvanishing of $F$ at the minimum means that the total superpotential VEV must be nonzero to cancel the global contribution to the vacuum energy in Eq. (3). The VEVs of the supersymmetry-preserving visible-sector fields may also shift from their globally determined values by small terms of order $O\left(m_{3 / 2}\right)$ and maintain zero value for their local F-terms. Special behavior is seen only for the components of the sliding multiplet $S$ which breaks supersymmetry.

Using the potential (3) in the pure BM case, we see that the field $S_{s}$ whose value was undetermined at the local/ metastable minimum of the global superpotential described above now picks up a potential which fixes its value to be of the order of the Planck scale. The other BM field $\phi_{s}$, which already had a large VEV [think of it as $\bar{\phi}_{s} \sim 10^{12} \mathrm{GeV}$ on phenomenological grounds in the context of the gravitymediated (SUGRY) models we are developing], will only experience a tiny shift in its VEV. To leading order we can determine the VEV $\left\langle S_{s}\right\rangle \equiv \bar{S}_{s}$ by fixing $\phi_{s}$ at its globally determined value $\bar{\phi}_{s}=-\mu_{B} / 2 \lambda_{B}$. The potential to be minimized is then

$$
\begin{aligned}
V\left(S_{s}\right) & =e^{\kappa^{2}\left|S_{s}\right|^{2}+\delta}\left\{\left(\delta \kappa^{2}\left|W_{0}+S_{s} \theta\right|^{2}+\left|\theta+\kappa^{2} S_{s}^{*}\left(W_{0}+S_{s} \theta\right)\right|^{2}\right.\right. \\
& \left.-3 \kappa^{2}\left|W_{0}+S_{s} \theta\right|^{2}\right\} .
\end{aligned}
$$

Here $\delta=\kappa^{2}\left(\left|\bar{\phi}_{s}\right|^{2}\right)$ is a tiny background fixed parameter $\left(\sim 10^{-12}\right)$ for the purposes of the extremization with respect to the globally undetermined VEV of $S_{s}$ and has essentially no effect on the value of $S_{s}$ at the extremum found. Defining dimensionless variables

$$
\begin{aligned}
x & =\kappa \frac{\widehat{W}_{0}}{\theta} ; & y & =\kappa S_{s}, \\
\varphi_{x} & =\operatorname{Arg}[x] ; & \varphi_{y} & =\operatorname{Arg}[y],
\end{aligned}
$$

the potential becomes

$$
\begin{aligned}
\widetilde{V} \equiv & \frac{V}{|\theta|^{2}}=\left\{\left(|x|^{2}+|y|^{2}\right)(\delta-3)+\left(1+|y|^{2}\right)^{2}+|x|^{2}|y|^{2}\right. \\
& \left.+2 \cos \left(\varphi_{y}-\varphi_{x}\right)|x||y|\left(|y|^{2}+\delta-2\right)\right\} .
\end{aligned}
$$

We emphasize that $x$ (which is the dimensionless form of the constant term in the superpotential) is not a dynamical variable but a parameter to be tuned to maintain the Minkowski vacuum energy at zero. Similarly, $\delta$ is a parameter fixed by the globally determined background to the $S_{s}$ extremization at a lower order of the expansion in powers of $\kappa$ and not a variable.

The only stable minimum of (7) with respect to $y$ (i.e. $S_{s}$ ) with zero energy (i.e., for the following, where field subscripts denote partial derivatives):

$$
V=V_{|y|}=V_{\theta_{y}}=0=V_{|y|, \theta_{y}} ; \quad V_{|y|,|y|}, V_{\theta_{y} \theta_{y}}>0
$$

is achieved as

$$
\begin{aligned}
\varphi_{y}=\varphi_{x} ; \quad x & =2-\sqrt{3-\delta} ; \quad y=y_{0}=\sqrt{3-\delta}-1, \\
\frac{\partial_{|y|} \partial_{|y|} \tilde{V}}{\partial_{\varphi_{y}} \partial_{\varphi_{y}} \tilde{V}} & =4 \sqrt{3-\delta} ;
\end{aligned}
$$


It is clear that the condition for a local minimum (2) is satisfied for

$$
\delta<3-\left(1+\sqrt{\left|\frac{\kappa^{2} \theta}{2 \lambda_{B}}\right|}\right)^{2} \simeq 2
$$

As mentioned, $\delta$ is tiny, and this condition is trivially satisfied. Thus, we have fixed the leading contribution $\bar{S}_{S}$ at the Planck scale by the combination of a gravitino-scale $\left(m_{3 / 2}=\kappa^{2}\langle W\rangle\right)$ mass and a tiny $\left[\sim\left(m_{3 / 2} / M_{\text {Planck }}\right)^{2}\right]$ quartic coupling for the sliding field $S_{s}$. The leading-order VEVs $\bar{\phi}_{s}, \bar{S}_{s}$ specify a Minkowski vacuum, with the degeneracy in $S_{s}$ lifted, to leading order in $\kappa \bar{\phi}_{s} \sim 10^{-13}$. There is no immediate reason to compute next-order shifts by perturbing around these VEVs, but it is straightforward to do so iteratively.

We now wish to build upon these complementary simple cases to first analyze a model in which a hidden-sector BM superpotential $W_{\mathrm{BM}}\left(S_{s}, \phi_{s}\right)=W_{H}$ with gauge singlets is coupled to a visible (GUT) sector (with fields $z_{i}$ ) which has a globally supersymmetric minimum associated with superpotential $W_{O}\left(z_{i}\right)$ and is invariant under a grand unifying gauge group broken spontaneously by the VEVs $\bar{z}_{i}$ at a superheavy scale $\left(M_{G} \sim 10^{16} \mathrm{GeV}\right)$ while preserving SUSY $[20,23,26]$. There is no coupling between the fields of the two sectors in the superpotential, so their global extrema and the nondetermination of $\bar{S}_{s}$ are unchanged. It is eminently reasonable to pursue the determination of the leading correction by setting fields $\phi, z_{i}$ already determined at the global level to those values $\left(\bar{\phi}, \bar{z}_{i}\right)$ and to determine the undetermined field (i.e. $S_{s}$ ) by minimizing (3) with respect to $S_{s}$, tuning $W_{0}$ to maintain zero energy at the vacuum. In this case, the entire discussion goes through verbatim with two superficial modifications:

(1) The constant term in the superpotential now also receives a contribution from the $\mathrm{VEV}$ of the visible sector, i.e. $W_{O}\left(\bar{z}_{i}\right)$.

(2) The tiny background-determined parameter $\delta$ now also contains the visible-sector values

$$
\delta=\kappa^{2}\left(\left|\bar{\phi}_{s}\right|^{2}+\sum_{i}\left|\bar{z}_{i}\right|^{2}\right) .
$$

Thus, the determination of $S_{s}$ is unchanged. The value of $\delta$ is still very small, since $M_{\mathrm{GUT}} \ll M_{\text {Planck }}, \delta<10^{-6}$. Condition (10) is still obviously trivially satisfied.

Finally, we arrive at the novel construction proposed in Ref. [26], where a (family) gauge symmetry links the BM hidden sector to the, via D terms, visible sector even at the global level, but there is still no link at the level of the superpotential. As we will see in the following sections, besides the field $S_{s}$, also the gauge nonsinglet parts $\hat{S}$ of the sliding field remain undetermined by the global F-term conditions. However, the visible-sector contributions to the family gauge group D-terms will not [26] vanish. In the BM sector, VEVs of the family nonsinglet part of $\phi$ field have zero $\mathrm{VEV}$, so that $\phi$ makes no contribution to the family Dterms, while the undetermined $\hat{S}$ nonsinglet BM VEVs will contribute to the positive definite family group D-terms. Since the visible contribution is $\sim M_{\mathrm{GUT}}^{4}$, it is obviously highly favorable for the $\hat{S}$ fields to take VEVs and cancel this positive definite contribution to the global SUSY potential. For definiteness, let the family gauge group be the $O\left(N_{g}\right), N_{g}=2,3$ actually used in Ref. [26] (for cogent structural and phenomenological reasons). Then an $O\left(N_{g}\right)$ gauge transformation can always align the family D-term VEV with any one generator of the gauge group and thus requires (in that aligned basis) only one $\hat{S}$ component to get a VEV. It is clear that there will be a pseudo-Goldstone degeneracy among the remaining $\hat{S}$ fields which may be lifted by supergravity effects. Since field $\hat{S}$ gets a VEV $\overline{\hat{S}} \ll M_{\text {Planck }}$, it too affects the $S_{s} \operatorname{VEV}$ (like $\bar{\phi}_{s}, \bar{z}_{i}$ ) only very weakly through $\delta$, which now takes the value

$$
\delta=\kappa^{2}\left(\left|\bar{\phi}_{s}\right|^{2}+\sum_{i}\left|\bar{z}_{i}\right|^{2}+\operatorname{Tr} \overline{\hat{S}}^{\dagger} \overline{\hat{S}}\right)
$$

and the rest of the determination of the BM-Polonyi field $S_{S}$ goes through unchanged.

As usual in gravity-mediated scenarios, the chiral fermion in the local supersymmetry-breaking $S$ multiplet is the global SUSY-breaking Goldstino that furnishes the longitudinal mode of the gravitino, which gets the mass

$$
m_{\frac{3}{2}}=\kappa^{2}\left|\sqrt{\bar{E}}\left(W_{0}+W_{O}\left(\bar{z}_{i}\right)+\overline{W_{H}}\right)\right| .
$$

Since this mass should be $\sim 10^{3}-10^{5} \mathrm{GeV}$ in typical gravity-mediated scenarios and typically $\bar{W}_{\mathrm{GUT}} \sim M_{X}^{3}>$ $10^{48} \mathrm{GeV}$, it is clear that $W_{0}$ must be used to cancel $\bar{W}_{\mathrm{GUT}}$ so that

$$
|\langle W\rangle| \simeq M_{p}|\theta| \simeq 10^{39}-10^{41} \mathrm{GeV}^{3} .
$$

The scale of supersymmetry breaking is fixed by the BM superpotential parameters:

$$
\sqrt{\left|F^{S}\right|}=\sqrt{|\theta|}=\left|\frac{\mu_{B}}{2 \sqrt{\lambda_{B}}}\right| \sim 10^{10.5}-10^{11.5} \mathrm{GeV}
$$

Provided $\left|\lambda_{B}\right|>10^{-14}$, one has $\left|\lambda_{B} S\right| \gg\left|\mu_{B}\right|$.

Having discussed the case $N_{g}=1$ in this section and indicated the general structure and solution strategy for our model [26], where family gauge symmetry-but not superpotential couplings - ties the visible and hidden sectors together, we will discuss the details of the global superpotentials and family representations in the next 
section for $N_{g}=2,3$ while the moduli mass spectra associated with the $\hat{S}$ "familion moduli" are discussed in Sec. IV.

A slightly subtle point that follows from the role of $\mathcal{F}$ as a local supersymmetry order parameter is that $z_{i}, \phi_{s}$ get small corrections of order $m_{3 / 2}$ so as to maintain $\mathcal{F}_{z_{i}, \phi_{s}} \equiv 0$, and similarly the family gauge nonsinglet BM fields $\hat{\phi}$ remain zero (see the next section) so that $\mathcal{F}_{\hat{\phi}} \equiv 0$. However since $\bar{S}_{s} \sim M_{\text {Planck }}, \overline{\hat{S}} \sim M_{\text {GUT }}$ while $\bar{W} \neq 0$, it is clear that $\mathcal{F}_{S_{s}, \hat{S}}$ are not zero but $\mathcal{F}_{\hat{S}} / \mathcal{F}_{S_{s}} \sim M_{\text {GUT }} / M_{\text {Planck }} \ll 1$, so that $S_{S}$ still dominates the supersymmetry breaking.

\section{GAUGED $O\left(N_{g}\right)$ AND BM VACUA}

Assuming that the visible-sector supersymmetry conditions are satisfied, the vanishing of the family symmetry D-terms for the GUT sector VEVs $\left(\bar{z}_{i}\right)$ alone is by no means guaranteed. In global SUSY, the $O\left(N_{g}\right)$ D-terms have the form

$$
\begin{aligned}
D_{O\left(N_{g}\right)}^{a} & =\operatorname{Tr}\left(\hat{\phi}^{\dagger}\left[T^{a}, \hat{\phi}\right]+\hat{S}^{\dagger}\left[T^{a}, \hat{S}\right]\right)+\bar{D}_{X}^{a}, \\
\bar{D}_{X}^{a} & =\sum_{i} \bar{z}_{i}^{\dagger} \mathcal{T}^{a} \bar{z}_{i},
\end{aligned}
$$

where $\bar{D}_{X}^{a}$ is the contribution of the visible-sector fields, and $T^{a}, \mathcal{T}^{a}$ are the generators of $O\left(N_{g}\right)$ in the fundamental and generic representations.

Since we wish to use a flat direction of the hidden-sector superpotential to cancel the contribution $\overline{D_{X}^{a}}$ of the visible sector, we consider the situation where $\phi_{s}, S_{s}$ become the trace modes of $O\left(N_{g}\right)$ symmetric representations $\phi_{a b}, S_{a b}$ so that the BM superpotential becomes

$$
W_{H}=\operatorname{Tr} S\left(\mu_{B} \phi+\sqrt{N_{g}} \lambda_{B} \phi^{2}\right) .
$$

Generically,

$$
S=\hat{S}+\frac{1}{\sqrt{N_{g}}} S_{s} \mathcal{I}_{N_{g}} ; \quad \operatorname{Tr} \hat{S}=0 .
$$

$\mathcal{I}_{N_{g}}$ is the $N_{g}$-dimensional unit matrix.

Before performing the case-wise analysis, consider the general features of the minimization of the Bajc-Melfo potential arising from the hidden-sector potential $W_{H}$ alone when $N_{g}>1$. The BM local minimum we find is characterized by an indeterminate VEV of all the fields $S_{S}, \hat{S}$ in the symmetric multiplet $S$, while the VEV of $\phi$ is fixed. The arguments given above and the evaluations in the next section show that the VEV of the family singlet in $S$ is determined by supergravity effects, while the others are fixed by minimizing the family D-terms [which are of $\left.O\left(M_{\mathrm{GUT}}^{4}\right)\right]$ together with the leading corrections to them, namely the supergravity induced soft mass terms
$\left[O\left(m_{3 / 2}^{2} M_{\mathrm{GUT}}^{2}\right)\right]$, all of which starts from considering the BM local supersymmetry-breaking minimum and its flat direction for $N_{g}>1$ together with the globally supersymmetric VEVs $\bar{z}_{i}$ as the background in which the determination of the sliding multiplet VEVs $S_{s}, \hat{S}$ are determined. We built up the argument for this fixation stepwise in Sec. II, and the novelty is now only the determination of the $\hat{S}$ VEVs.

The F-terms arising from $N_{g}$ are

$$
\begin{aligned}
& F_{\phi}=\mu_{B} S+\lambda \sqrt{N_{g}}(S . \phi+\phi . S), \\
& F_{S}=\mu_{B} \phi+\lambda \sqrt{N_{g}} \phi . \phi .
\end{aligned}
$$

Clearly the associated global superpotential

$$
V(\phi, S)=\operatorname{Tr}\left(F_{S}^{\dagger} F_{S}+F_{\phi}^{\dagger} \cdot F_{\phi}\right)
$$

is semipositive definite. We search for the generalization of the BM local minimum by the ansatz

$$
\langle\phi\rangle=\frac{1}{\sqrt{N_{g}}} \bar{\phi}_{s} \mathcal{I}_{N_{g}}
$$

We emphasize that this implies that the gauge nonsinglet $\operatorname{VEV} \hat{\phi} \equiv 0$ for all $N_{g}$. In fact, this feature implies that after the introduction of supergravity corrections $\mathcal{F}_{\hat{\phi}}=0$ [unlike $\mathcal{F}_{\hat{S}}$, which develops a value $O\left(m_{3 / 2} M_{\mathrm{GUT}}\right)$ and thus makes a $M_{\mathrm{GUT}} / M_{\text {Planck }}$ suppressed contribution to the Goldstino]. With the ansatz (21), then,

$$
\begin{aligned}
& \left\langle F_{\phi}\right\rangle=\langle S\rangle\left(\mu_{B}+2 \lambda \bar{\phi}_{s}\right), \\
& \left\langle F_{S}\right\rangle=\left\langle\bar{\phi}_{s}\right\rangle\left(\mu_{B}+\lambda \bar{\phi}_{s}\right) \mathcal{I}_{N_{g}},
\end{aligned}
$$

so that $\phi_{s}=-\mu_{B} /(2 \lambda)$ still ensures that $\left\langle F_{\phi}\right\rangle,\left\langle F_{\hat{S}}\right\rangle$ vanish provided $\langle\phi\rangle$ has the form in (21). Moreover, the fluctuation $\tilde{\phi}$ around this VEV receives a positive definite mass squared

$$
V=4\left|\lambda\left\langle S_{s}\right\rangle\right|^{2} \operatorname{Tr} \tilde{\phi}^{\dagger} \tilde{\phi}+\cdots .
$$

from the VEV of the singlet in $S_{s}$, which is undetermined by the BM potential but fixed by supergravity corrections to be of order $M_{p}$ as shown above. These contributions dominate all others. On the other hand,

$$
\left\langle F_{S}\right\rangle=-\frac{\mu_{B}^{2}}{4 \lambda} \mathcal{I}_{N_{g}}
$$

breaks supersymmetry and causes corrections of order $\pm \mu_{B}^{2}$ to the scalar masses of the $\phi$ supermultiplet. We again emphasize that the F-term of $\hat{S}$ vanishes for the BM 
minimum for all $N_{g}$. The mass spectrum is discussed in detail in Section IV.

\section{A. Determination of $S_{ \pm}$when $N_{g}=2$}

For $N_{g}=2$ it is convenient to use the isomorphism $O(2) \simeq U(1)$ and define

$S=\frac{1}{2}\left(\begin{array}{cc}\sqrt{2} S_{s}+S_{+}+S_{-} & i\left(S_{-}-S_{+}\right) \\ i\left(S_{-}-S_{+}\right) & \sqrt{2} S_{s}-\left(S_{+}+S_{-}\right)\end{array}\right)$,

and similarly for $\phi=\left[\phi_{a b}\right]$, where $S_{ \pm}, S_{s}$ are properly normalized fields so that $\operatorname{Tr} S^{\dagger} S=S_{+}^{\dagger} S_{+}+S_{-}^{\dagger} S_{-}+S_{s}^{\dagger} S_{s}$ and $\operatorname{Tr} S \phi=S_{s} \phi_{s}+S_{(+} \phi_{-}$. In this notation an $O(2)$ vector $\psi_{a}$ has charges $\pm 1 / 2: \psi_{ \pm 1 / 2}=\left(\psi_{1} \pm i \psi_{2}\right) / \sqrt{2}$. The superpotential becomes

$$
\begin{aligned}
W_{H}= & \mu_{B}\left(S_{s} \phi_{s}+S_{(+} \phi_{-)}\right) \\
& +\lambda_{B}\left(S_{s} \phi_{s}^{2}+2 S_{s} \phi_{+} \phi_{-}+2 \phi_{s} S_{(+} \phi_{-}\right),
\end{aligned}
$$

and it is easy to check that $\bar{\phi}_{s}=-\mu_{B} / 2 \lambda_{B}, \bar{\phi}_{ \pm}=0$ solves the F-term conditions for $W_{H}$ (all F-terms vanish except that $\overline{\partial_{S_{s}} W}=\theta$ as before). This verifies that the general form of the VEVs of $\phi, F_{S}$ is indeed that given in (21) and (22). The fields $S_{s, \pm}$ remain undetermined at this local minimum, and the superpotential reduces to the one for $N_{g}=1$ when the fields that are determined are equated to their values at the local minimum. Notice that the VEV $\bar{\phi}$ makes the coefficient of $S_{(+} \phi_{-)}$vanish so that there is no Dirac mass mixing the chiral fermions in $S_{ \pm}, \phi_{\mp}$.

The calculation for the singlet goes through unchanged fixing $S_{s} \sim M_{p}$ as in Eqs. (21) and (22). With this VEV, the $O(2)$ charged fermionic components of $\hat{\phi}_{ \pm}$get masses $\sim \lambda_{B} \bar{S}_{s}$. Along with the GUT-scale breaking of the family symmetry, the large mass of the modes in $\phi_{a b}$ is responsible for quelling the percolation of the supersymmetry breaking coded in $F_{S_{s}}$ and ensuring the hidden sector is actually hidden. Since $\hat{\phi}_{ \pm}$have zero VEVs at the SUSY-breaking minimum, they do not mix with the family symmetry gaugino. Since the charged $S$ fields can have VEVs of at most the order of the GUT scale $M_{\mathrm{GUT}} \ll M_{P}$, the potential for their determination to leading order in $m_{3 / 2}$ is simply the common scalar mass term generated by the supergravity potential and the D-term of the family symmetry. In the $O(2)$ case, the leading-order potential for the flat family charged $S$ directions is

$$
\begin{aligned}
V\left(S_{+}, S_{-}\right)= & m_{3 / 2}^{2}\left(\left|S_{+}\right|^{2}+\left|S_{-}\right|^{2}\right) \\
& +\frac{g_{f}^{2}}{2}\left(\left|S_{+}\right|^{2}-\left|S_{-}\right|^{2}+\bar{D}_{X}\right)^{2}
\end{aligned}
$$

here $\bar{D}_{X}=\sum q_{i}\left|\bar{z}_{i}\right|^{2}$, where $q_{i}, \bar{z}_{i}$ are the family symmetry charges of the global supersymmetric VEVs in the visible sector. Clearly, if $x=\operatorname{Sign}\left[D_{X}\right]$, the minimum will occur when

$$
S_{-x}=\sqrt{\left|\bar{D}_{X}\right|-x \frac{m_{3 / 2}^{2}}{g^{2}}} ; \quad \bar{S}_{x}=0 .
$$

Notice that the shift in the $S_{-x}$ VEV relative to $\sqrt{\bar{D}_{X}}$ is tiny, but the gravity induced mass term does enforce the choice of which component of $\hat{S}$ gets a VEV. The vacuum energy after minimization is $\sim m_{3 / 2}^{2}\left|D_{X}\right|$ and can be tuned to zero by a shift $\delta \hat{W}_{0} \sim m_{3 / 2}\left|D_{X}\right|<m_{3 / 2} M_{p}^{2}$. Note that since $\bar{F}_{\hat{S}}=0$ from (26) (and $\bar{F}_{z_{i}}=0$ by our scenario), the supergravity corrections to the global D-terms shown in (3) are zero.

\section{B. Fixation of S VEVs for $N_{g}=3$}

For $N_{g}=3$ we define the components of $S_{a b}$ in terms of $T_{3}$ eigenfields to be

$$
\left(\begin{array}{ccc}
\frac{1}{6}\left(\sqrt{6} S_{0}+3 i S_{-2}-3 i S_{+2}+2 \sqrt{3} S_{s}\right) & \frac{1}{2}\left(S_{+2}+S_{-2}\right) & \frac{1}{2}\left(S_{+}+S_{-}\right) \\
\frac{1}{2}\left(S_{-2}+S_{+2}\right) & \frac{1}{6}\left(\sqrt{6} S_{0}-3 i S_{-2}+3 i S_{+2}+2 \sqrt{3} S_{s}\right) & \frac{1}{2} i\left(S_{+}-S_{-}\right) \\
\frac{1}{2}\left(S_{+}+S_{-}\right) & \frac{1}{2} i\left(S_{+}-S_{-}\right) & \frac{1}{\sqrt{3}}\left(S_{s}-\sqrt{2} S_{0}\right)
\end{array}\right),
$$

and similarly for $\phi=\left[\phi_{a b}\right] . S_{s, 0, \pm, \pm 2}$ are properly normalized fields, so that

$$
\begin{aligned}
\operatorname{Tr} S^{\dagger} S & =S_{+2}^{\dagger} S_{+2}+S_{-2}^{\dagger} S_{-2}+S_{+}^{\dagger} S_{+}+S_{-}^{\dagger} S_{-}+S_{s}^{\dagger} S_{s}, \\
\operatorname{Tr} S \phi & =S_{s} \phi_{s}+S_{(+} \phi_{-)}+S_{(+2} \phi_{-2)} .
\end{aligned}
$$

In this case the explicit form of the BM superpotential is [where (square) round brackets denote (anti-)symmetrization] 


$$
\begin{aligned}
W_{\mathrm{BM}}= & \mu_{B}\left(S_{s} \phi_{s}+S_{0} \phi_{0}+S_{(+} \phi_{-)}+S_{(+2} \phi_{-2)}\right)+\lambda_{B}\left[\left(S_{s}\left(\phi_{s}^{2}+\phi_{0}^{2}+2 \phi_{-} \phi_{+}+2 \phi_{-2} \phi_{+2}\right)\right.\right. \\
& +S_{0}\left(-\phi_{0}^{2} / \sqrt{2}+2 \phi_{s} \phi_{0}-\sqrt{2} \phi_{-} \phi_{+}+\sqrt{2} \phi_{-2} \phi_{+2}\right)+2 S_{(+} \phi_{-)} \phi_{s}-\frac{1}{\sqrt{2}} S_{(+} \phi_{-)} \phi_{0}+i \sqrt{3}\left(S_{+} \phi_{-2} \phi_{+}-S_{-} \phi_{-} \phi_{+2}\right) \\
& \left.+\sqrt{2} S_{(+2} \phi_{-2)} \phi_{0}+2 S_{(+2} \phi_{-2)} \phi_{s}+\frac{i}{2} S_{[+2} \phi_{-2]}^{2}\right]
\end{aligned}
$$

Using this one may again verify that the VEVs are of the form given in Eqs. (21) and (22).

When $N_{g}=3$, the D-terms form a vector of $O(3)$, and it is advantageous to change to a basis (denoted by primes) where $\bar{D}_{X}^{\prime a}=\delta_{3}^{a}\left|\bar{D}_{X}\right|$. This is easily achieved by a rotation in the 12 plane to rotate the $\bar{D}_{X}^{a}$ vector into the 23 plane, and then a 23 plane rotation to make it point in the 3 direction:

$$
\begin{aligned}
O & =R_{23}\left[\theta_{X}\right] \cdot R_{12}\left[\frac{\pi}{2}-\varphi_{X}\right], \\
\theta_{X} & =\operatorname{ArcTan}\left[\sqrt{\left.\frac{V_{1}^{2}+V_{2}^{2}}{V_{3}^{2}}\right] ; \quad \varphi_{X}=\operatorname{ArcTan}\left[\frac{V_{2}}{V_{1}}\right],},\right.
\end{aligned}
$$

where $V^{a}=\bar{D}_{X}^{a}$. The potential for the flat directions from $\hat{S}^{\prime}$ is now

$$
\begin{aligned}
V\left[\hat{S}^{\prime}\right]= & m_{3 / 2}^{2}\left(\left|S_{0}^{\prime}\right|^{2}+\left|S_{+}^{\prime}\right|^{2}+\left|S_{-1}^{\prime}\right|^{2}+\left|S_{+2}^{\prime}\right|^{2}+\left|S_{-2}^{\prime}\right|^{2}\right) \\
& +\frac{g_{f}^{2}}{2}\left\{\left(\left|S_{+}^{\prime}\right|^{2}+2\left|S_{+2}^{\prime}\right|^{2}-\left|S_{-}^{\prime}\right|^{2}-2\left|S_{-2}^{\prime}\right|^{2}+\left(\bar{D}_{X}^{3}\right)^{\prime}\right)^{2}\right. \\
& \left.+2 \operatorname{Tr}\left(S^{\prime \dagger}\left[T_{+}, S^{\prime}\right]\right) \operatorname{Tr}\left(S^{\prime \dagger}\left[T_{-}, S^{\prime}\right]\right)\right\} .
\end{aligned}
$$

This has a solution along the same lines as for the $N_{g}=2$ case. Dropping primes, one gets

$$
\begin{aligned}
\left|\bar{S}_{-2}\right| & =\sqrt{\frac{\left|\vec{D}_{X}\right|}{2}-\frac{m_{3 / 2}^{2}}{4 g_{f}^{2}}}, \\
\bar{S}_{-,+,+2} & =0 .
\end{aligned}
$$

\section{MASS SPECTRUM}

The spectrum from the visible-sector gauge interactions and superpotential is sensible by assumption and worked out for realistic cases in Ref. [26]. Our interest here is in the BM fields and - where relevant - their mixing with visiblesector fields through gauge or supergravity terms. We shall confine ourselves to working out the leading contributions and ignore the effects of small shifts $\left(\bar{z}_{i} \rightarrow \bar{z}_{i}+\delta_{i}\right.$, where $\delta_{i}$ of order $m_{3 / 2}$ ) in visible-sector VEVs which we assume [via the usual shift $\delta_{i}=-\left(W^{\prime \prime}\right)_{i j}^{-1} \bar{z}^{j} \kappa^{2} \bar{W}$ ] maintain $\mathcal{F}_{z_{i}}=0$.
We first consider the mass terms that arise in the globally supersymmetric Lagrangian when the fields $\phi_{s}, z_{i}, S_{s}, \hat{S}$ are replaced by their VEVs. The supergravity corrections are necessarily suppressed by the Planck length and are therefore important only if a field does not obtain a mass at one of the large scales present, $\left\{M_{P}, M_{\mathrm{GUT}}, \sqrt{M_{P} M_{3 / 2}} \gg\right.$ $\left.m_{3 / 2}\right\}$. At the global level, VEVs of F-terms do not couple to fermion fields at all, so that any SUSY-breaking contributions will be visible only in the scalar sector. Since only $F_{S_{s}} \neq 0$, it follows that it makes a SUSYbreaking mass contribution of order $\bar{F}_{S_{s}} \sim m_{3 / 2} M_{P}$ to the scalars that it couples to in the cubic terms of the superpotential.

In the family gauge terms (we ignore the visible gauge sector under which the BM fields are singlets) the (global SUSY-preserving) $\hat{S}$ VEVs mix the chiral fermion in the $\hat{S}$ multiplet with the visible-sector chiral fermion combination that couples to the family gauginos to form a Dirac multiplet. Since $\hat{S}$ terms otherwise lack mass terms, this leads to a residual massless fermion from the orthogonal mode. This is explained more explicitly below.

In the supergravity Lagrangian, only fermion bilinears coupling with nonvanishing $\mathcal{F}$ terms can possibly obtain mass terms sensitive to supersymmetry breaking. Only $\mathcal{F}_{S_{s}} \sim m_{3 / 2} M_{\text {Planck }}$ can be nonzero at leading order, although the $\mathcal{F}_{z_{i}, \hat{S}}$ will get smaller contributions of order $m_{3 / 2} M_{\text {GUT }}$.

\section{A. Mass spectrum for $N_{g}=1$}

In this case only gravity couples the visible and hidden sectors. The corrections to the visible sector are the familiar soft supersymmetry-breaking supergravity corrections which are insensitive to the detailed form of the hiddensector superpotential or whether its SUSY-breaking minimum is local or global. The VEV $\bar{\phi}_{s}=-\mu_{B} / 2 \lambda_{B}$ implies that the field $S_{s}$ (which has no mass term $W=m_{S} S_{s}^{2}+\cdots$ ) does not get a mass by mixing with $\phi_{s}$ and provides the Goldstino as expected, even though the supersymmetry is broken by a local rather than a global minimum. The fermion masses consist of the $\phi_{s}$ mode with mass $2 \lambda_{B} \bar{S}_{s}$ and the gravitino with longitudinal mode from the Goldstino $\tilde{S}_{s}$.

In the BM sector, the only fermion mass terms at the global level are 


$$
-\mathcal{L}=\lambda_{B} \bar{S}_{s} \tilde{\phi}_{s} \tilde{\phi}_{s}=\frac{m_{\tilde{\phi}_{s}}}{2} \tilde{\phi}_{s} \tilde{\phi}_{s} .
$$

Mass terms due to the VEV $\bar{\phi}_{s}$ cancel. The scalar mass spectrum consists of two real scalar modes from $\phi_{S}$ with masses squared $\left|2 \lambda_{B} \bar{S}\right|^{2} \pm\left|\mu_{B}\right|^{2} / 2$ (notice the supersymmetry-breaking splitting of the scalars from the chiral fermion's Majorana mass $2 \lambda_{B} \bar{S}_{s}$ ) and two real scalars from $S_{s}$ with masses squared $m_{3 / 2}^{2}((7+4 \sqrt{3}) \pm(5+3 \sqrt{3}))$. This completes the discussion of the $N_{g}=1$ case apart from explicitly working out the effective theory, which will be of the usual supergravity type (global SUSY GUTderived MSSM plus soft SUSY-breaking terms).

\section{B. Mass spectrum for $N_{g}=2$}

Consider the masses of the superfields $\hat{\phi}_{a b}$. The fermions do not couple directly to the SUSY-violating F-term. Inserting the VEVs in the fermion bilinear terms obtained from the superpotential in Eq. (26), we get the following fermion mass terms only (tildes here denote fermionic components):

$$
-\mathcal{L}=\lambda_{B} \bar{S}_{s}\left(\tilde{\phi}_{s} \phi_{s}+2 \tilde{\phi}_{+} \tilde{\phi}_{-}\right)+2 \lambda_{B} \tilde{\phi}_{s} \tilde{\phi}_{(-} \bar{S}_{+)} .
$$

Since $\bar{S}_{s} \gg \bar{S}_{ \pm}$, it is clear that the $\tilde{\phi}_{s} \tilde{\phi}_{ \pm}$mixing is far subdominant to the diagonal Majorana and Dirac mass terms $\tilde{\phi}_{s}^{2}, \tilde{\phi}_{+} \tilde{\phi}_{-}$. Since there is no residual gauge group, the chiral mass matrix is just $\overline{\partial_{i} \partial_{j} W}$. We have a symmetric $3 \times 3$ chiral mass matrix with rows and columns labeled by $\left\{\phi_{s}, \phi_{+}, \phi_{-}\right\}$:

$$
m_{\phi \phi}=\lambda_{B} \bar{S}_{s}\left(\begin{array}{ccc}
1 & \epsilon & 0 \\
\epsilon & 0 & 1 \\
0 & 1 & 0
\end{array}\right),
$$

where $\epsilon=\bar{S}_{-x} / \bar{S}_{s} \sim M_{X} / M_{p}$. This can easily be diagonalized and gives large masses in the GUT-scale range for reasonable values $\lambda_{B} \sim 10^{-3}$ to $10^{-1}$. In the scalar sector the $\phi$ fields also receive mass-squared contributions from $\lambda_{B} F_{S_{s}} \sim \mu_{B}^{2}$ besides the contributions shown for the fermions. However, since $\left|\mu_{B}\right| \ll M_{X} \ll M_{p}$, these will lead to small splitting of the dominant contributions shared with the fermions. All this is a straightforward extension of the behavior of the BM $\phi$ for $N_{g}=1$.

With the VEVs given in Sec. III for $N_{g}=2$, and due to the lack of any other mass term for $S_{ \pm}$, it is easy to check that the fermionic component of $S_{-x}$ mixes with the visiblesector Higgsino field combination that combines to form a Dirac multiplet with the single family gaugino of the $O(2)$ gauge symmetry, because the visible-sector Higgs fields carry symmetric representation of the family group. Thus, the Dirac partner $\Lambda$ for the $U(1)_{f}$ gaugino $\lambda_{f}$ with Dirac mass

$$
m_{\lambda_{B}}=\sqrt{2} g_{f} \sqrt{\left|\bar{S}_{-s}\right|^{2}+\sum_{i} q_{i}^{2}\left|\bar{z}_{i}\right|^{2}}
$$

is defined by

$$
\begin{aligned}
\mathcal{L} & =-m_{\lambda} \Lambda \lambda_{g}+\text { H.c., } \\
\Lambda & =\left(\cos \left[\theta_{S}\right] \tilde{Z}-\sin \left[\theta_{S}\right] \tilde{S}_{-x}\right) \frac{\sqrt{2} g_{f}}{m_{\lambda_{B}}} \\
\tilde{Z} & =\frac{\sum_{i} q_{i} \bar{z}_{i}^{*} \tilde{z}_{i}}{\sqrt{\sum_{i} q_{i}^{2}\left|\bar{z}_{i}\right|^{2}}}, \\
\tan \theta_{S} & =\frac{x\left|\bar{S}_{-x}\right|}{\sqrt{\sum_{i} q_{i}^{2}\left|\bar{z}_{i}\right|^{2}}} .
\end{aligned}
$$

However, due to the absence of other mixing terms for $S_{ \pm s}$, the orthogonal combination $\tilde{\chi}$ and $S_{x}$ remain massless at leading order. Here

$$
\tilde{\chi}=\cos \left[\theta_{S}\right] \tilde{S}_{-x}+\sin \left[\theta_{S}\right] \tilde{Z}
$$

does not mix with the remaining massive modes, since it is made of (SM neutral) zero modes $\tilde{S}_{-x}, \Lambda$ of the chiral mass matrices in the hidden and visible sectors, respectively. Shifts due to supergravity are too small to shift the masses $m_{S_{+} \phi_{-}}=m_{\phi_{+} S_{-}}=\mu_{B}+2 \lambda \bar{\phi}_{s}=0$ appreciably. So the leading contributions come from loop effects (see Fig. 1) (analogous to the one-loop corrections to neutrino masses induced by soft SUSY breaking [30]) and have value

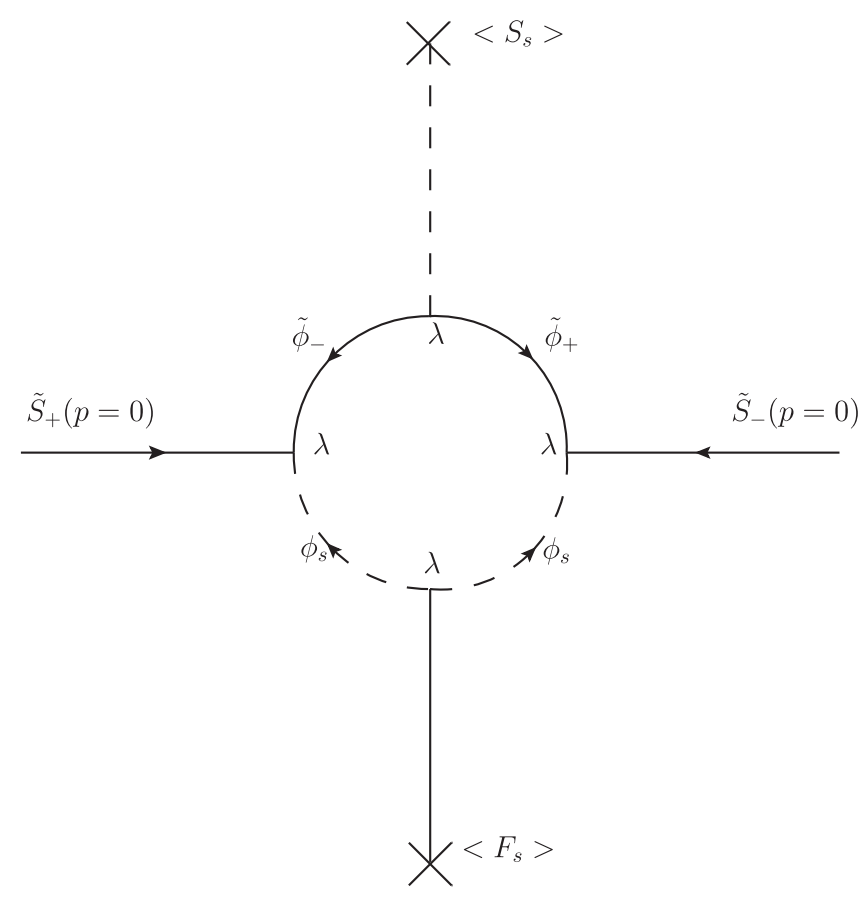

FIG. 1. Diagram for fermionic mass $m_{\tilde{S}}$. 


$$
m_{\hat{S}}^{1 \text {-loop }} \simeq \frac{\left|\lambda_{B}\right|^{2}}{16 \pi^{2}} \frac{\bar{F}_{S}}{\bar{S}_{S}} \simeq \frac{\left|\lambda_{B}\right|^{2}}{16 \pi^{2}} \frac{m_{3 / 2}}{\sqrt{3}-1} .
$$

Thus, these masses may be as small as a few $\mathrm{GeV}$ even for a large gravitino mass. Due to their weak interactions with light modes, they may be suitable candidates for light cold dark matter. The scalar partners have masses

$$
\left(m^{2}\right)_{S_{x}}=2 m_{3 / 2}^{2} ; \quad m_{\operatorname{Re}\left(S_{-x}\right)}^{2}=2 g_{f}^{2}\left|D_{X}\right|-2 m_{3 / 2}^{2} .
$$

Like $\tilde{S}_{-x}$, the hidden-sector Goldstone scalar component $\operatorname{Im}\left[S_{-x}\right]$ will again lead to a nearly massless mode, since it too will mix with the $O(2)$ Goldstone contributions from the visible sector, and hence the orthogonal mode (partner of the pseudo-Goldstino $\tilde{\chi}$ ) will be massless before loop effects. The scalar pseudo-Goldstone mode $\chi$ which remains massless even after symmetry breaking due to pseudo-doubling of Goldstone modes (i.e. independent breaking of the family symmetry in the potentials from the unlinked hidden- and visible-sector superpotentials) can also obtain loop induced mass from the partner of the graph shown for the fermions, but it will remain light. This is because the supersymmetry-breaking F-term couples to other particles through $\phi_{a b}$ propagators whose large masses $\lambda_{B}\left\langle S_{s}\right\rangle \sim M_{X}$ ensure that the effect of one-loop corrections is a power of $F / M_{X} \sim m_{3 / 2} M_{p} / M_{X}$. It is clear that since all the other modes have large masses much greater than the gravitino mass, and we have already taken into account the dominant contributions to the fields that participate in the SUSY breaking, the supergravity corrections to their masses are subdominant and of no immediate significance to warrant their presentation here.

\section{Mass spectrum for $N_{g}=\mathbf{3}$}

The analysis for this case proceeds in a manner closely parallel to the case of two generations. Once again, all contributions from the VEV of $\phi_{s}$ cancel, leaving for the $\mathrm{BM}$ fermion mass terms only

$$
\begin{aligned}
-\mathcal{L}= & \lambda_{B} \bar{S}_{s}\left(\tilde{\phi}_{s} \tilde{\phi}_{s}+\tilde{\phi}_{0} \tilde{\phi}_{0}+2 \tilde{\phi}_{+} \tilde{\phi}_{-}+2 \tilde{\phi}_{+2} \tilde{\phi}_{-2}\right) \\
& +2 \lambda_{B} \tilde{\phi}_{s} \tilde{\phi}_{+2} \bar{S}_{-2} .
\end{aligned}
$$

Once again we have diagonal Majorana/Dirac masses $2 \lambda_{B} \bar{S}_{s}$ for all the components of $\phi$ and a much smaller offdiagonal contribution from the VEV of the nonsinglet $\hat{S}$. These will be accompanied by scalar masses of the same magnitude up to small splitting $O\left(m_{3 / 2}\right)$. Similarly, the scalars from the $S_{s}$ multiplet will obtain masses of the order of the gravitino mass.

As for the $N_{g}=2$ case, fermionic partners of the $\hat{S}$ particles (except $S_{-2}$, but even so, the analog of $\tilde{\chi}$ will remain light via the mechanism explained for the $N_{g}=2$ case) do not get mass to leading order. The Dirac partners of the family symmetry gauginos in the primed basis, where $\bar{D}^{a} \sim \delta_{3}^{a}$, are as follows:

$$
\begin{aligned}
\Lambda^{3} & =\frac{-2{\overline{S^{\prime}}}_{-2}^{\dagger}{\tilde{S^{\prime}}}_{-2}+\sum_{i} q_{i} \bar{z}_{i}^{\prime \dagger} \tilde{z}_{i}^{\prime}}{\left(4{\overline{S^{\prime}}}_{-2}{\overline{S^{\prime}}}_{-2}+\sum_{i} \bar{q}_{i}^{2} z_{i}^{\prime \prime} \bar{z}_{i}^{\prime}\right)^{1 / 2}}, \\
\Lambda^{ \pm} & =\frac{\sum_{i} \bar{z}_{i}^{\prime \dagger} \mathcal{T}^{ \pm} \tilde{z}_{i}^{\prime}}{\left(\sum_{i} \bar{z}_{i}^{\prime \dagger} \mathcal{T}^{+} \mathcal{T}^{-} \bar{z}_{i}^{\prime}\right)^{1 / 2}} .
\end{aligned}
$$

As before, the fermion mode orthogonal to $\Lambda^{3}$ will remain massless along with $\tilde{S}_{0, \pm, 2}$.

The scalar masses are positive (apart from the Goldstone boson)

$$
\begin{aligned}
M_{S_{0}}^{2} & =m_{3 / 2}^{2} ; & M_{S_{-}}^{2} & =\frac{1}{2} m_{3 / 2}^{2}, \\
M_{S_{+}}^{2} & =\frac{3}{2} m_{3 / 2}^{2} ; & M_{S_{+2}}^{2} & =2 m_{3 / 2}^{2}, \\
M_{R_{-2}}^{2} & =4 g_{f}^{2} \mid \frac{\left|\vec{D}_{X}\right|}{2}-2 m_{3 / 2}^{2} ; & M_{I_{-2}}^{2} & =0 .
\end{aligned}
$$

In the above, since we are working in the primed basis, we have exhibited only the Goldstone longitudinal component of the $O(3)$ gauge boson in the 3 direction which comes from the field $S_{-2}$ : of course, all three $O\left(N_{g}\right)$ massive gauge bosons will get longitudinal mode contributions from the visible sector (which we assume breaks the family symmetry completely) which we have not discussed explicitly here [see, however, Ref. [26] for more details in a concrete model based on a realistic $S O(10)$ GUT]. Since only a linear combination of $I_{-2}$ and a visible-sector massless mode will be eaten, we would again expect the orthogonal combination to remain massless.

Now we will have five light fermionic and one scalar degree of freedom lighter than the gravitino mass and with very weak couplings to ordinary matter. Again they will pick up mass at one loop due to supersymmetry breaking and will remain as the fossils of flavor and supersymmetry breaking. Thus, they are either candidates for multicomponent relic dark matter or must be removed somehow.

From the above discussion, it is clear that the BM SUSY-breaking Minkowski vacuum is characterized by the presence of several light fields coming from the supersymmetry-breaking $\left(S_{s}\right)$ and from the $O\left(N_{g}\right)$ variant $\hat{S}$ fields over and above the usual MSSM light fields in the visible sector. The superfields $\left(\phi_{a b}\right)$ are superheavy, with masses of order $\lambda_{B} \bar{S}_{S} \sim M_{X}$. The effective low-energy theory must be written in terms of both sets of light fields by equating the heavy fields to their VEVs and separating the light fields out via an expansion in $m_{3 / 2} / M_{p}$. This calculation proceeds largely like that in Ref. [5], but care must be taken due to the additional gauge invariance active in both hidden and gauge sectors, the main effects of which the present analysis sought to clarify. 
It bears mention that radiative effects in such systems may be quite significant and have an important bearing on cosmological behavior [31]. The loop-corrected Kahler potential is available up to two loops [32]. We will return to the study of the vacuum and cosmological predictions in the theory with both gravity and radiative effects in a sequel.

\section{DISCUSSION}

In this paper we have shown how gravity mediation of Bajc-Melfo-type calculable supersymmetry breaking at metastable vacua in a hidden sector containing fields variant under a family gauge symmetry ensures the viability of GUT and family symmetry breaking in the visible sector of a "grand Yukawonification" [26] model. In such models, one aims at generating the observed hierarchical fermion flavor structure from a gauged family symmetry model with only generation blind couplings. The special role of the BM supersymmetry breaking is that it provides flat directions in both the $O\left(N_{g}\right)$ singlet and the gauge variant parts of a symmetric chiral supermultiplet $S_{a b}=S_{b a}$. Since it is very difficult to make the contribution of the visiblesector GUT fields to the $O\left(N_{g}\right)$ D-terms vanish, the $\hat{S}$ flat direction performs the invaluable function of canceling this contribution without disturbing the symmetry breaking in the visible sector. The determination of the viability of the "grand Yukawonification" model then becomes a matter of searching the relatively small remaining parameter space for viable parameter sets that fit the fermion data at $M_{X}$ while taking account of threshold corrections at low and high scales and while respecting constraints on crucial quantities like the proton lifetime $[20,23,24,25]$. Note that in this approach, not only are the hard parameters of the visible-sector superpotential reduced by replacement of the flavored parameters by bland family symmetric ones, but also the soft supersymmetry-breaking parameters are determined by the two parameters of the hidden-sector superpotential and the Planck scale.

The structure used entails yet further stringent constraints, since the masslessness of the moduli multiplets $\hat{S}_{a b}$ before supersymmetry breaking implies the existence of $N_{g}\left(N_{g}+1\right) / 2-1$ SM singlet fermions generically lighter than the gravitino mass scale and possibly as light as a few GeV. In addition, the Polonyi mode $S_{s}$ may also lead to difficulties in the cosmological scenario. Thus, such modes can be both a boon and a curse for familion GUT models. A boon because generic SUSY GUT models are hard put if asked to provide SUSY WIMPs of mass below $100 \mathrm{GeV}$ as CDM candidates, as suggested by the DAMA/ LIBRA experiment [28]. A curse because there are strong constraints on the existence of such light moduli which normally demand that their mass be rather large ( $>10 \mathrm{TeV}$ ) due to the robust cosmological ("Polonyi") problems arising from decoupled modes with Planck scale VEVs [29]. In contrast to the simple Polonyi model and String moduli, the BM moduli have explicit couplings to light fields through family D-term mixing and loops. Moreover, the MSGUT scenario favors [23,25] large gravitino masses $>5-50 \mathrm{TeV}$. Thus, the Polonyi and moduli problems may be evaded. In any case, the cosmology need be considered seriously only after we have shown that the MSSM fermion spectrum is indeed generated by the "Yukawonified" NMSGUT [26].

\section{ACKNOWLEDGMENTS}

I am very grateful to Borut Bajc for numerous valuable discussions. I thank Charanjit K. Khosa for collaboration and Ila Garg for discussions and help with preparing the manuscript. I acknowledge my employment at the Physics Department, Panjab University Chandigarh at the commencement of this work and the hospitality of the CERN theory group during its submission for publication.
[1] E. Cremmer, S. Ferrara, L. Girardello, and A. Van Proeyen, Yang-Mills theories with local supersymmetry: Lagrangian, transformation laws and super-Higgs effect, Nucl. Phys. B212, 413 (1983).

[2] N. Ohta, Grand unified theories based on local supersymmetry, Prog. Theor. Phys. 70, 542 (1983).

[3] A. H. Chamseddine, R. L. Arnowitt, and P. Nath, Locally Symmetric Grand Unification, Phys. Rev. Lett. 49, 970 (1982); history and exhaustive original references in R. Arnowitt, A. H. Chamseddine, and P. Nath, The development of supergravity grand unification: Circa 1982-85, Int. J. Mod. Phys. A 27, 1230028 (2012); The development of supergravity grand unification: Circa 1982-85, Int. J. Mod. Phys. A 27, 1292009(E) (2012).
[4] S. Weinberg, Does Gravitation Resolve the Ambiguity Among Supersymmetry Vacua? Phys. Rev. Lett. 48, 1776 (1982).

[5] L. J. Hall, J. D. Lykken, and S. Weinberg, Supergravity as the messenger of supersymmetry breaking, Phys. Rev. D 27, 2359 (1983).

[6] J. Polony, Budapest preprint No. KFKI-1977-93 (1977) (unpublished).

[7] L. O'Raifeartaigh, Spontaneous supersymmetry breaking for chiral scalar superfields, Nucl. Phys. B96, 331 (1975).

[8] S. Dimopoulos, G. R. Dvali, R. Rattazzi, and G. F. Giudice, Dynamical soft terms with unbroken supersymmetry, Nucl. Phys. B510, 12 (1998). 
[9] K. Intriligator, N. Seiberg, and D. Shih, Dynamical SUSY breaking in metastable vacua, J. High Energy Phys. 04 (2006) 021.

[10] M. A. Luty, Simple gauge-mediated models with local minima, Phys. Lett. B 414, 71 (1997); K. Agashe, GUT and SUSY breaking by the same field, Phys. Lett. B 444, 61 (1998); Improved GUT and SUSY breaking by the same field, Nucl. Phys. B588, 39 (2000).

[11] B. Bajc and A. Melfo, Metastable gauged O'Raifeartaigh, J. High Energy Phys. 04 (2008) 062.

[12] S. Ray, Some properties of metastable supersymmetrybreaking vacua in Wess-Zumino models, Phys. Lett. B 642, 137 (2006).

[13] E. Witten, Mass hierarchies in supersymmetric theories, Phys. Lett. 105B, 267 (1981).

[14] B. A. Ovrut and S. Raby, The geometrical hierarchy model and $N=1$ supergravity, Phys. Lett. 125B, 270 (1983).

[15] C. S. Aulakh, Local supersymmetry and hi-lo scale induction, Report No. CCNY-HEP-83/2; , Ph.D. thesis, CCNY, 1983, Report No. UMI-84-01477, http://physics.puchd.ac .in/aulakh/PhDthesis.

[16] C. S. Aulakh and R. N. Mohapatra, Report No. CCNY-HEP82-4, 1982; Report No. CCNY-HEP-82-4-REV, 1982; Implications of supersymmetric $\mathrm{SO}(10)$ grand unification, Phys. Rev. D 28, 217 (1983).

[17] T. E. Clark, T. K. Kuo, and N. Nakagawa, An SO(10) supersymmetric grand unified theory, Phys. Lett. 115B, 26 (1982).

[18] C. S. Aulakh, B. Bajc, A. Melfo, G. Senjanovic, and F. Vissani, The minimal supersymmetric grand unified theory, Phys. Lett. B 588, 196 (2004).

[19] B. Bajc, A. Melfo, G. Senjanovic, and F. Vissani, The minimal supersymmetric grand unified theory. I: Symmetry breaking and the particle spectrum, Phys. Rev. D 70, 035007 (2004).

[20] C. S. Aulakh and A. Girdhar, $S O(10)$ MSGUT: Spectra, couplings and threshold effects, Nucl. Phys. B711, 275 (2005).
[21] C.S. Aulakh, MSGUTs from germ to bloom: Towards falsifiability and beyond, arXiv:hep-ph/0506291.

[22] C. S. Aulakh and S. K. Garg, MSGUT: From bloom to doom, Nucl. Phys. B757, 47 (2006).

[23] C.S. Aulakh and S. K. Garg, The new minimal supersymmetric GUT : spectra, RG analysis and fermion fits, Nucl. Phys. B857, 101 (2012).

[24] C. S. Aulakh, NMSGUT III: Grand unification upended, arXiv:1107.2963.

[25] C. S. Aulakh, I. Garg, and C. K. Khosa, Baryon stability on the Higgs dissolution edge: Threshold corrections and suppression of baryon violation in the NMSGUT, Nucl. Phys. B882, 397 (2014).

[26] C. S. Aulakh and C. K. Khosa, $S O(10)$ grand unified theories with dynamical Yukawa couplings, Phys. Rev. D 90, 045008 (2014).

[27] C. S. Aulakh and A. Girdhar, $S O(10)$ a la Pati-Salam, Int. J. Mod. Phys. A 20, 865 (2005).

[28] R. Bernabei, P. Belli, S. d'Angelo, A. Di Marco, F. Montecchia, F. Cappella, A. d'Angelo, A. Incicchitti et al., Dark matter investigation by DAMA at Gran Sasso, Int. J. Mod. Phys. A 28, 1330022 (2013).

[29] G. D. Coughlan, W. Fischler, E. W. Kolb, S. Raby, and G. G. Ross, Cosmological problems for the Polonyi potential, Phys. Lett. 131B, 59 (1983); B. de Carlos, J. A. Casas, F. Quevedo, and E. Roulet, Model independent properties and cosmological implications of the dilaton and moduli sectors of 4D strings, Phys. Lett. B 318, 447 (1993).

[30] C. S. Aulakh and R. N. Mohapatra, Supersymmetry and the calculation of neutrino masses, Phys. Lett. 121B, 147 (1983).

[31] M. Dine, W. Fischler, and D. Nemeschansky, Phys. Lett. 136B, 169 (1984).

[32] For two-loop results and references to earlier one-loop results, see S. Groot Nibbelink and T. S. Nyawelo, Two-loop effective Kahler potential of (non-)renormalizable supersymmetric models, J. High Energy Phys. 01 (2006) 034. 